\title{
Granulocyte Therapy for Cancer
}

\author{
Wenying Wang * and Dong Wang $\dagger \ddagger^{\Delta}$
}

GRANULOCYTE THERAPY FOR CANCER is the first book ever published dealing with the origin and development of the Granulocyte Therapy for Cancer. Dr. Zheng Cui has been regarded as "the father of cancer resistance super-mice", made great contribution to the progress of Cancer Immunology and Immunotherapy. This book, based on his ten years' research, intends to present a comprehensive overview from the serendipity discovery of the cancer complete resistant/ spontaneous regression (CR/SR) mice to the relevant cancer target killing mechanism mediated by leukocytes, as well as how the innovative therapeutic approach, Leukocyte Infusion Therapy (LIFT) applicable to human cancer.

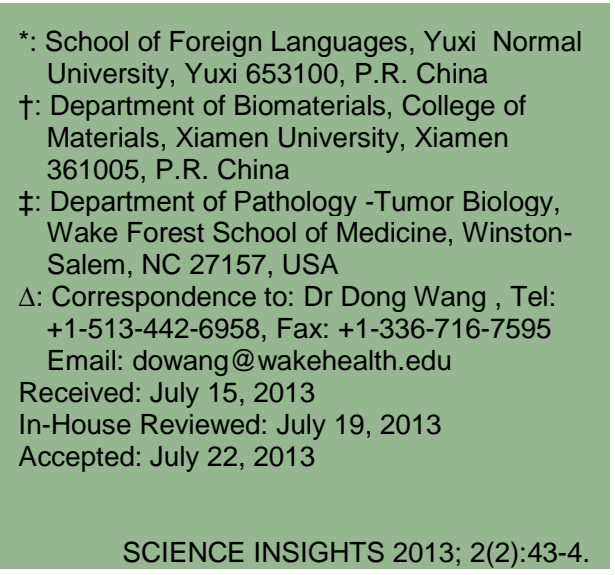

\section{Keywords: Cancer - Granulocyte Therapy - Immunity - Mortality}

$\mathrm{I}$ MMUNE SYSTEM is the most important defense system to protect the host from disease. Strong evidence showed that immune system also plays an important role in protecting against cancer. However, how the immune system works in defending and fighting against cancer and the underlying molecular mechanisms are still unknown.

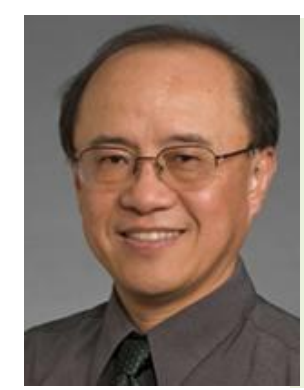

Granulocyte Therapy for Cancer, which originated in a serendipity discovery of a cancer complete resistant/ spontaneous regression $(\mathrm{CR} / \mathrm{SR})$ "super-mouse", presents a comprehensive description of the tenyear research about the cancer killing mechanism in the "super-mice" family and the following clinical tests of the Leukocyte Infusion Therapy (LIFT) approach. The book contains eleven chapters that are sequentially organized into five topics including the overview of etiology, cytology and development of cancer treatment, historic perspective of cancer immune surveillance, the discovery and following research of the $\mathrm{CR} / \mathrm{SR}$ "super-mice" family, bacterial infection and cancer regression and the LIFT clinical test on patient.

glycolysis, larger amount of lactic acid metabolite, a large number of negatively charges on cell surface, failure of or damaged function of mitochondria, dysfunction of fat metabolism, a big increase in the amount of protein degradation en-

Zheng Cui, M.D. Ph.D., is a biochemist and oncologist currently serving as an Associate Professor of Pathology (Tumor Biology) at Wake Forest University in Winston-Salem, USA. Based on several years' research on cancer resistance or CR/SR "super-mice" family and clinical trials, Dr. Cui has proposed a unique and still controversial idea that certain individuals naturally produce a special kind of granulocyte that contains an inherent resistance or super killing activity to cancer. These granulocytes could potentially be extracted from donors and given to cancer victims to fight against cancer. This novel experimental therapy, named Leukocyte Infusion Therapy (LIFT), has been approved by FDA to practice the phase I and phase II clinical study..

The book starts with an overview on cancer and the current methods of anticancer treatment. The author put emphasis on the causes of death from malignant tumor and liquid cancer, and different opinions on some controversial issues in cancer research field, such as the tumor stem cell, the treatment of cancer by inducing apoptosis etc were presented. Eight characteristics of cancer cells are concentrated on at the end of the chapter: extremely unstable genome, easier for the glucose uptake, greater ability to tolerate hypoxia and the anaerobic tus of cancer research.

In the chapter 2, a brief historical overview and some typical events like the exploration of the relationship between the immune system and cancerous disease were presented. From the well-known "Coley's toxins" (the primary immunotherapy approach for cancer) to the hypothesis of "cancer surveillance" and to "cancer immune surveillance” (proposed by Paul Ehrlich and Sir MacFarlane Burnet - 1908 and 1960 Nobel Prize laureates in Physiology or Medicine, respectively), the author pointed out 
that the experimental and clinical evidence supported the potential effect of immune system, particularly the lymphocytes, on the cancer immune surveillance, however, the possible contribution from other leukocyte populations was ignored. Meanwhile, the author also gave a concise description about the fundamental difference between the innate immunity and adaptive immunity. At the end of this part, the author proposed that the poor curative effect of the current clinical immunotherapy for cancer by stimulating the activities of $\mathrm{T}$ and $\mathrm{B}$ lymphocytes and natural killer cells may result from an irreversible damage of the immune function in cancer patients. Finally, the chapter was concluded with two questions: 1) Is there a cancer specific immune surveillance system in healthy body, while this system no longer exists or be damaged in cancer patients? 2) What is the exact immune component serving this cancer specific surveillance system?

Chapter 3 discussed the serendipity discovery of the $\mathrm{SR} / \mathrm{CR}$ "super mouse", which was the only survivor of a study in which mice were given injections of S180 cancer cells. Since then, thousands of mice have been bred from the original SR/CR mouse and approximately 30 percent of the offspring inherited the cancer SR/CR trait, in an autosomal manner. The author gave a detailed description about the confirmed anti-cancer mechanism from which it showed that the cancer resistance in SR/CR mice was mediated by the special immune system, exactly like the innate immune system with neutrophils, macrophages, and natural killer cells. The results supporting the innate immunity in cancer resistance were provided in detail, and three phases: infiltration, tight contact and tumor destruction were also explained. Moreover, you can find accompanying figures helping you conveying these concepts. In the chapters 4 and 5 , in order to normalize cancer resistance ability, an in vitro assay of Cancer Killing Activity (CKA) has been developed and introduced. The author described the CKA assay in SR/CR mouse phenotype test, and then in human granulocyte assay. At last, the prospect of transfusing "super strength" cancer-killing granulocyte from donors to boost the cancerkilling ability of patient was discussed.

The chapter 6 entitled "Effect mechanisms of granulocyte and targeting" and the chapter 7 entitled "Cancer cells are the target of activated granulocyte" highlighted the mechanism of granulocyte activation

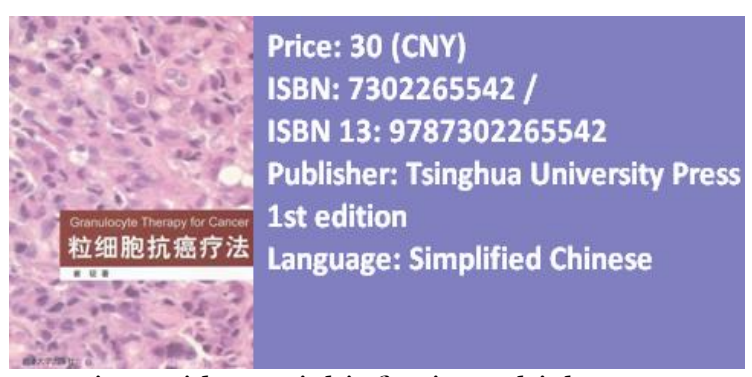

in anti-bacterial infection which carried out mainly by the human neutrophil peptides (HNP) and the unique surface charge property of cancer cells, from which cancerous cells become the target of the activated granulocytes.

The last part (chapters 8, 9 and 10) provided the prospect of clinical application of Leukocyte Infusion Therapy (LIFT) for cancer. The author firstly proposed a hypothesis, as same as the cancer resistant mice, that some cancer resistant human exist, and they had "super strength" cancerkilling leukocyte during their entire lives. Then, the author gave a sketch about the development and current situation of traditional adoptive immunotherapy for cancer including lymphokine-activated killer cell (LAK) therapy, tumor infiltrating lymphocytes (TIL) therapy and cytokine-induced killers (CIK) therapy. At the end, the LIFT means, which has been approved by FDA to implement the phase I and phase II clinical trials, was highlighted and briefly introduced as follow: volun- teers who will be selected as donors based on the observed potential CKA of their granulocytes will complete the leukapheresis blood donation to collect their "super strength" granulocytes. The cancer patients then will receive the granulocytes through a transfusion. This was considered as a truly innovative therapeutic approach possessing fundamental difference with the existing immunotherapy. In further, problems of this therapy were also provided, such as 1) how to collect and reserve sufficient "super strength" cancer-killing granulocytes? 2) whether patients can tolerate the transfused granulocytes? 3) which types of cancer would be more suitable for this treatment? These are the questions need to be answered, and also they are future directions for research

In sum, it has been more than ten years since the discovery of the SR/CR "super mouse", and many significant advances have been made, particularly with regard to the ongoing clinical trials of the Leukocyte Infusion Therapy which originated from this "super mouse". Therefore, the current book is necessary for a very diverse audience. The whole book is written succinct meaningful for being appeal to professionals and non-professionals. This is the first book of its kind and is a must-to-beread for professionals, researchers, clinicians and students who are interested in the field of cancer immunology and specific granulocyte immune therapy.

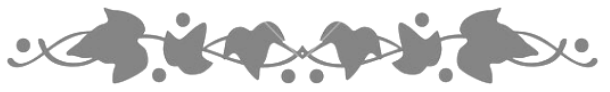

SCIENCE INSIGHTS ISSN: 2329-5856 www.bonoi.org 